\title{
New improvements of HASTA for the analysis of chomospheric solar events
}

\author{
L. Leuzzi ${ }^{1,2}$, C. Francile ${ }^{1,2}$, M. L. Luoni ${ }^{3}$, M. Rovira ${ }^{3}$ \\ and J. I. Castro ${ }^{1,2}$ \\ ${ }^{1}$ Facultad de Ciencias Exactas, Físicas y Naturales (FCEFyN), Universidad Nacional de San \\ Juan (UNSJ), San Juan, Argentina \\ email: luisleuzzi@yahoo.com.ar \\ ${ }^{2}$ Félix Aguilar Astronomical Observatory (OAFA), UNSJ, San Juan, Argentina \\ email: cfrancile@unsj-cuim.edu.ar \\ ${ }^{3}$ Instituto de Astronomía y Física del Espacio (IAFE), Argentina \\ email: mluoni@iafe.uba.ar
}

\begin{abstract}
It is well known that chromospheric observations in the hydrogen alpha line give relevant information about solar flares, plages and protuberances, among other typical features of the Sun. From 1998 to 2006, the HAlpha Solar Telescope of Argentina (HASTA) has provided solar images to the scientific community with the technological resources available at that time. Starting in 2007, major improvements have been incorporated, like a new CCD camera with enhanced spatial and temporal resolution, filter replacement, the automatic focusing system, and a new flat-fielding procedure. The hardware changes also called for software improvements, and a new solar-flare classification routine was implemented. At present, the Félix Aguilar Observatory (OAFA) of the University of San Juan (UNSJ) has a permanent staff of observers which now permits continuous solar monitoring. We expect that all these advances will allow to analyze chromospheric solar activity, especially solar flares, in more detail.
\end{abstract}

Keywords. Instrumentation: adaptive optics - Sun: chromosphere - Sun: flares - Sun: filaments Sun: activity - waves - telescopes

\section{Introduction}

The HASTA telescope, located 50km eastwards from the Andes mountains at an altitude of $2370 \mathrm{~m}$, has been operating continuously since 1998 at the Carlos U. Cesco Station (EACUC), El Leoncito, San Juan, Argentina as part of an agreement for scientific cooperation involving three institutions: Max Planck Institut für Extraterrestrische Physik (MPE) of Germany, and Instituto de Astronomía y Física del Espacio (IAFE) and "Félix Aguilar" Observatory (OAFA) the last two from Argentina, to observe the Solar Chromosphere (Bagalá et al. 1999). During the past two years new instrumental and software developments have been implemented to the telescope carried out by OAFA (Francile et al. 2008), to improve and extend its observational capabilities of the dynamics of solar atmosphere and solar activity.

\section{Characteristics of the instrument and its improvements}

The place where the telescope is located is extremely low air humidity and without human activity that might cause atmospheric pollution.

HASTA is a solar refractor telescope that provides full sun CCD images in the Hydrogen $\mathrm{H} \alpha$ emission line at $656.3 \mathrm{~nm}$. Table 1 shows the main features of the telescope. 


\begin{tabular}{lcc}
\hline Telescope & Diameter & $11 \mathrm{~cm}$ \\
& Focal Distance & $165 \mathrm{~cm}$ \\
\hline Location & Longitude & $69.3 \mathrm{~W}$ \\
& Latitude & $31.8 \mathrm{~S}$ \\
& Coverage of Time & $11: 30 \mathrm{UT}$ to $20: 30 \mathrm{UT}$ \\
\hline Filter & Type & Tunable Lyot - Öhmann \\
& Central Wavelengh & $656.27 \mathrm{~nm}$ \\
& Bandwidth & $0.03 \mathrm{~nm}$ \\
& Tuning Range & $\pm 0.11 \mathrm{~nm}$ \\
\hline Images & Normal Size & $(1280 \times 1024)$ pixels - Full Sun Disk \\
& Integration Time & $50-100 \mathrm{msec}$ \\
& Type & Center Line \& Line Wings, sequential \\
& Resolution & $\approx 2$ arc sec \\
\hline Operational Modes & Patrol Mode & Images every $\approx 3 \mathrm{~min}$ \\
& Flare Mode & Images every $0.5 \mathrm{sec}$ \\
\hline
\end{tabular}

Table 1. Main characteristics of the HASTA

The solar activity is analyzed in real time and, if a fast event is detected, the camera switches into high-speed mode or Flare mode. In this mode it can take and store fullframe images up to 2 per second during the Impulsive Phase of a Flare.

The New CCD Camera. The images are registered with a PCO-1600 CCD Camera with 512 Mbytes internal memory allowing high speed and resolution recording. The typical exposure times are 50-100 milliseconds (see the Table 1).

The New Flat Fielding Procedure. A new procedure to obtain a flat-field image was developed using a diffuser of the type holographic $60^{\circ}$. Some flat field images are generated daily by means of the new diffuser placed at the entrance of the telescope. These images are pre-processed and median averaged to reduce the statistic noise to get a better image for the flat field correction.

Automatic Focusing System. The continuous focus misalignments of the telescope due to the daily thermal variations that modifies its mechanical properties and the physical properties of the air inside it, are automatically corrected by the HASTA control software.

This software analyzes the spectral components of the measured intensities on the sun disk and limb, and obtains a focusing factor. As this factor is affected by the local conditions at the telescope place (clouds, seeing, weather) and also by the solar activity conditions (flares, plages, filaments), a feedback loop is needed to maintain the focus factor as optimum as can be possible for these conditions during the whole day. This action is performed moving a focusing stepper motor recently installed. In this way the quality of the sun observations are improved considerably.

The New Controlled Tuning Device for the "Lyot-Öhman" H $\alpha$ Filter. HASTA takes full-disk images as $\mathrm{H} \alpha$ line center as wings. By means of a software the scientist can define automatic wavelength-shift sequences for the tunable $\mathrm{H} \alpha$ filter. It allows to control the motors that rotate the input and output filter polarizers to achieve the desired frequency shift. 

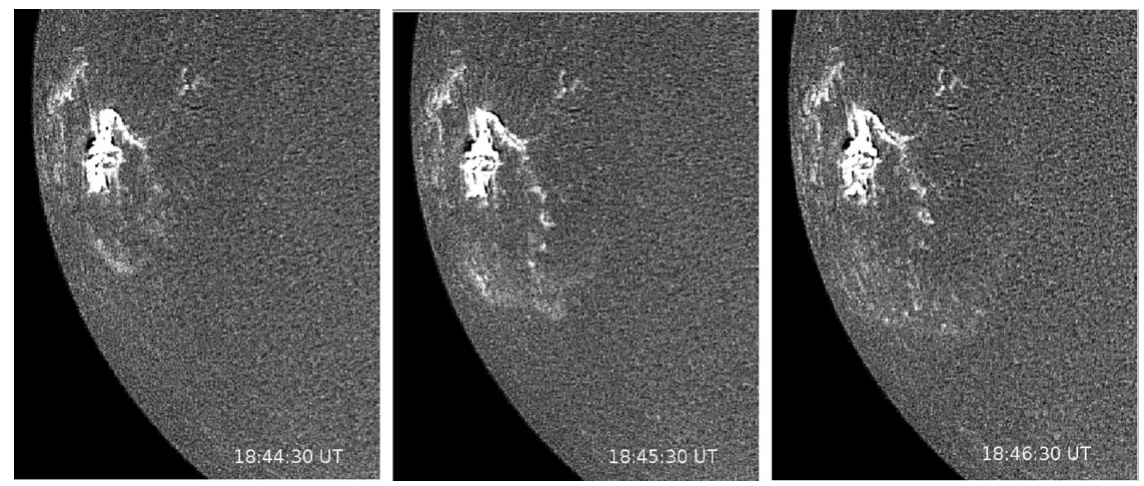

Figure 1. Sequence of $\mathrm{H} \alpha$ images shows a tsunami-like shock wave spreading from a class X6.5 flare erupting from AR 10930 on 2006 December 06. The contrast and brightness of the images have been enhanced to bring up details of the shock wave incoming.

\section{Example of observation of a Moreton Wave}

The Moreton waves are shock wave, they are large arch-shaped brightenings propagating through the Sun observed in $\mathrm{H} \alpha$. They are observed immediately following large solar flares (Athay \& Moreton 1960). By other side, although such flares do occur there are not reports of Moreton waves. Some recent studies (Cliver et al. 1999) suggest that Moreton waves may originate from fast coronal mass ejections.

These images are a solar full-disk fraction and were recorded on 2003 December 06 at $\mathrm{H} \alpha$ line center $(656.27 \mathrm{~nm})$ with a cadence of $0.5 \mathrm{sec}$ (pixel size $=2 ")$. We note that the observations show the details of the expanding Moreton wave as an intensity enhancement.

\section{Conclusions}

HASTA represents an important tool, both for solar physics and space weather research, which will certainly increase our knowledge about the physical processes behind the various chromospheric phenomena and their interrelations.

In this part of Southern hemisphere this solar telescope is the only one with such high spatial and temporal resolution. All the improvements taken part in the HASTA enable to follow the evolution of rapid changing phenomena like flares and propagating waves as well as filaments, in the solar chromosphere.

\section{References}

Athay, R. \& Moreton, G. 1961, ApJ, 133, 935

Bagalá, G., Bauer, O., Fernández Borda, R., Francile, C., Haerendel, G., \& Rovira, M. 1999, Proceedings of the 9th European Meeting on Solar Physics, ESA-SP, 448, 469

Cliver, E., Webb, D., \& Howard, R. 1991, Sol. Phys., 187, 89

Francile, C., Castro, J. I., Leuzzi, L., Luoni, M. L., Rovira, M. G., Cornudella, A., Gomez, W., \& Sarmiento, R. 2008, Bol. Asoc. Argen. Astr., 51, 339 